\title{
A majority of uninfected adults show preexisting antibody reactivity against SARS-CoV-2
}

\author{
Abdelilah Majdoubi, ${ }^{1,2}$ Christina Michalski,, ${ }^{1,2}$ Sarah E. O'Connell, ${ }^{3}$ Sarah Dada, ${ }^{1,2}$ Sandeep Narpala, ${ }^{3}$ \\ Jean Gelinas,, ${ }^{4,5}$ Disha Mehta, ${ }^{4,6}$ Claire Cheung,, ${ }^{1,2}$ Dirk F.H. Winkler, ${ }^{7}$ Manjula Basappa, ${ }^{3}$ \\ Aaron C. Liu,, ${ }^{1,2,8}$ Matthias Görges, ${ }^{1,6}$ Vilte E. Barakauskas, ${ }^{9}$ Mike Irvine, ${ }^{1}$ Jennifer Mehalko, ${ }^{10}$ \\ Dominic Esposito, ${ }^{10}$ Inna Sekirov, ${ }^{9,11}$ Agatha N. Jassem, ${ }^{9,11}$ David M. Goldfarb, ${ }^{1,2,12}$ Steven Pelech, ${ }^{7,13}$ \\ Daniel C. Douek, ${ }^{3}$ Adrian B. McDermott, ${ }^{3}$ and Pascal M. Lavoie ${ }^{1,2}$ \\ 'BC Children's Hospital Research Institute, Vancouver, British Columbia, Canada. ${ }^{2}$ Department of Pediatrics, University \\ of British Columbia, Vancouver, British Columbia, Canada. ${ }^{3}$ Vaccine Research Center, National Institute of Allergy and \\ Infectious Diseases, NIH, Bethesda, Maryland, USA. ${ }^{4}$ Department of Anesthesiology, Surrey Memorial Hospital (SMH), \\ Surrey, British Columbia, Canada. ${ }^{5}$ Department of Anesthesiology \& Pain Medicine, University of Alberta, Edmonton, \\ Alberta, Canada. ${ }^{6}$ Department of Anesthesiology, Pharmacology and Therapeutics, University of British Columbia, \\ Vancouver, British Columbia, Canada. ${ }^{7}$ Kinexus Bioinformatics Corporation, Vancouver, British Columbia, Canada. ${ }^{8}$ Vaccine \\ Evaluation Centre, BC Children's Hospital Research Institute, Vancouver, British Columbia. ${ }^{9}$ Department of Pathology and \\ Laboratory Medicine, University of British Columbia, Vancouver, British Columbia, Canada. ${ }^{10}$ National Cancer Institute RAS \\ Initiative, Cancer Research Technology Program, Frederick National Laboratory for Cancer Research, Leidos Biomedical \\ Research Inc., Frederick, Maryland, USA. "British Columbia Centre for Disease Control (CDC) Public Health Laboratory, \\ Vancouver, British Columbia, Canada. ${ }^{12}$ Division of Medical Microbiology, Department of Pathology and Laboratory \\ Medicine, and ${ }^{13}$ Department of Medicine, University of British Columbia, Vancouver, Canada.
}

Preexisting cross-reactivity to SARS-CoV-2 occurs in the absence of prior viral exposure. However, this has been difficult to quantify at the population level due to a lack of reliably defined seroreactivity thresholds. Using an orthogonal antibody testing approach, we estimated that about $\mathbf{0 . 6 \%}$ of nontriaged adults from the greater Vancouver, Canada, area between May 17 and June 19 , 2020, showed clear evidence of a prior SARS-CoV-2 infection, after adjusting for false-positive and false-negative test results. Using a highly sensitive multiplex assay and positive/negative thresholds established in infants in whom maternal antibodies have waned, we determined that more than $90 \%$ of uninfected adults showed antibody reactivity against the spike protein, receptorbinding domain (RBD), N-terminal domain (NTD), or the nucleocapsid (N) protein from SARSCoV-2. This seroreactivity was evenly distributed across age and sex, correlated with circulating coronaviruses' reactivity, and was partially outcompeted by soluble circulating coronaviruses' spike. Using a custom SARS-CoV-2 peptide mapping array, we found that this antibody reactivity broadly mapped to spike and to conserved nonstructural viral proteins. We conclude that most adults display preexisting antibody cross-reactivity against SARS-CoV-2, which further supports investigation of how this may impact the clinical severity of COVID-19 or SARS-CoV-2 vaccine responses.

owner of Kinexus Bioinformatics Corporation.

Copyright: (c) 2021, Majdoubi et al. This is an open access article published under the terms of the Creative Commons Attribution 4.0 International License.

Submitted: November 23, 2020

Accepted: March 12, 2021

Published: March 15, 2021

Reference information: JCI Insight 2021;6(8):e146316.

\section{Introduction}

Coronavirus disease 2019 (COVID-19) was declared a global pandemic on March 11, 2020, and has resulted in almost 100 million confirmed cases and 2.1 million deaths worldwide as of January 24, 2021. Almost all individuals infected with SARS-CoV-2 seroconvert within 2-3 weeks, with the spike and nucleocapsid (N) proteins eliciting the strongest responses $(1,2)$. While much attention has focused on defining immune reactivity in individuals after infection, other data indicate that many individuals show preexisting SARSCoV-2 cross-reactive T and B cells without prior exposure to the virus (3-5). However, the extent of preexisting SARS-CoV-2 antibody reactivity at the population level has been difficult to estimate, due to a lack 
of assay sensitivity (6) and clearly definable background thresholds to identify meaningful seroreactivity among individuals who have been unexposed to the virus (7).

There are 4 circulating coronaviruses predating COVID-19 that cause up to $30 \%$ of seasonal upper respiratory tract infections (8). The spike proteins of $\beta$-coronaviruses HKU1 and OC43 exhibit approximately 40\% sequence similarity, whereas the $\alpha$-coronaviruses NL63 and 229E exhibit approximately 30\% structural similarity with SARS-CoV-2 (9). The common occurrence of circulating coronaviruses year after year and their structural similarity with SARS-CoV-2 raises the possibility that the former may stimulate cross-reactive responses toward SARS-CoV-2 and that this heterotopic immunity may impact clinical susceptibility to COVID-19 and/or modulate responses to the SARS-CoV-2 vaccine $(10,11)$.

The main objective of this study was to estimate the extent of the preexisting seroreactivity against SARS-CoV-2 in the general adult population and its relationship to circulating coronaviruses. To confirm that SARS-CoV-2 antibody reactivity in uninfected adults was genuinely cross-reactive and not due to widespread unreported, asymptomatic SARS-CoV-2 circulation, we similarly assayed sera collected prior to the emergence of SARS-CoV-2 and from infants before and after maternal antibodies have waned. In addition, we used a SPOT peptide array to map this antibody reactivity on the SARS-CoV-2 proteome.

\section{Results}

Study population. In total, 276 healthy adults were recruited for this cohort between May 17 and June 19, 2020. The demographic characteristics and geographical area of residence of participants are shown in Supplemental Table 1 (supplemental material available online with this article; https://doi.org/10.1172/ jci.insight.146316DS1) and Supplemental Figure 1, respectively. The majority ( $n=196 ; 71 \%)$ were health care workers. Less than half had traveled outside of British Columbia (BC) since January 1,2020, to the USA, Europe, Iran, the Caribbean, Australia, Mexico and Japan. Two individuals had a history of PCR-confirmed COVID-19.

Prevalence of prior SARS-CoV-2 infection in the study population. To estimate the proportion of individuals who had been previously infected with SARS-CoV-2, we used a multiplex assay to profile antibody reactivity against 4 viral antigens: the whole SARS-CoV-2 spike protein, its N-terminal domain (NTD) and receptor-binding domain $(\mathrm{RBD})$, and the $\mathrm{N}$ protein. Clustering analysis based on antibody reactivity for these 4 antigens identified that 3 individuals (CW087, CW0150, FH0037) and 5 control sera from convalescent COVID-19 patients (controls A, B, C, D and E) clustered together, separately from the rest of the cohort (Figure 1). The antibody reactivity profile of these 8 distinct sera showed high reactivity against all 4 SARS-CoV-2 antigens, whereas all other individuals showed variable antibody reactivity against either spike, RBD, or the N protein (Supplemental Figure 2).

The 3 individuals (CW087, CW0150, FH0037) who clustered with the 5 control sera included the 2 individuals who had a history of PCR-confirmed COVID-19, plus an asymptomatic woman who was not aware she had COVID-19 initially but later identified that she had been in contact with a COVID-19 case about 90 days prior to serology testing for this study (Supplemental Table 2).

All sera from the cohort who displayed above-the-mean antibody reactivity for at least 1 of the 4 SARSCoV-2 antigens (i.e., for a total of 222 out of 276 individuals) were further tested with a commercial diagnostic commercial chemiluminescent (CLIA) assay, which recognizes the spike protein S1 antigen (Supplemental Figure 3). With this assay, the same 3 individuals (CW087, CW0150, FH0037), plus the 5 control sera mentioned above, tested positive. Therefore, based upon these data, it appeared that 3 of 276 participants $(1.1 \%)$ showed clear evidence of a previous infection with SARS-CoV-2. After adjusting for bias by using point estimates of specificity and sensitivity of the CLIA assay, we estimated that the prevalence of a previous SARS-CoV-2 infection was $0.60 \%(95 \% \mathrm{CI}, 0 \%-2.71 \%)$ in this cohort.

Antibody reactivity to circulating coronaviruses. The multiplex assay also included quantification of antibody reactivity against the spike proteins of circulating coronaviruses (OC43, HKU1, NL63, 229E). All individuals showed high antibody reactivity against the spike proteins from these circulating coronaviruses (Supplemental Figure 2). We used correlation analyses to understand the relationship between the antibody reactivity against SARS-CoV-2 and circulating coronaviruses. Among the 273 seronegative individuals, we detected significant correlations between antibody reactivity to SARS-CoV-2 spike, as well as spike proteins from HKU1, NL63, and 229E, but not OC43 (Supplemental Table 3 and Supplemental Figure 4).

Specificity of SARS-CoV-2 antibody reactivity in uninfected individuals. Next, we conducted competition experiments to exclude the possibility that the antibody reactivity against SARS-CoV-2 in uninfected 


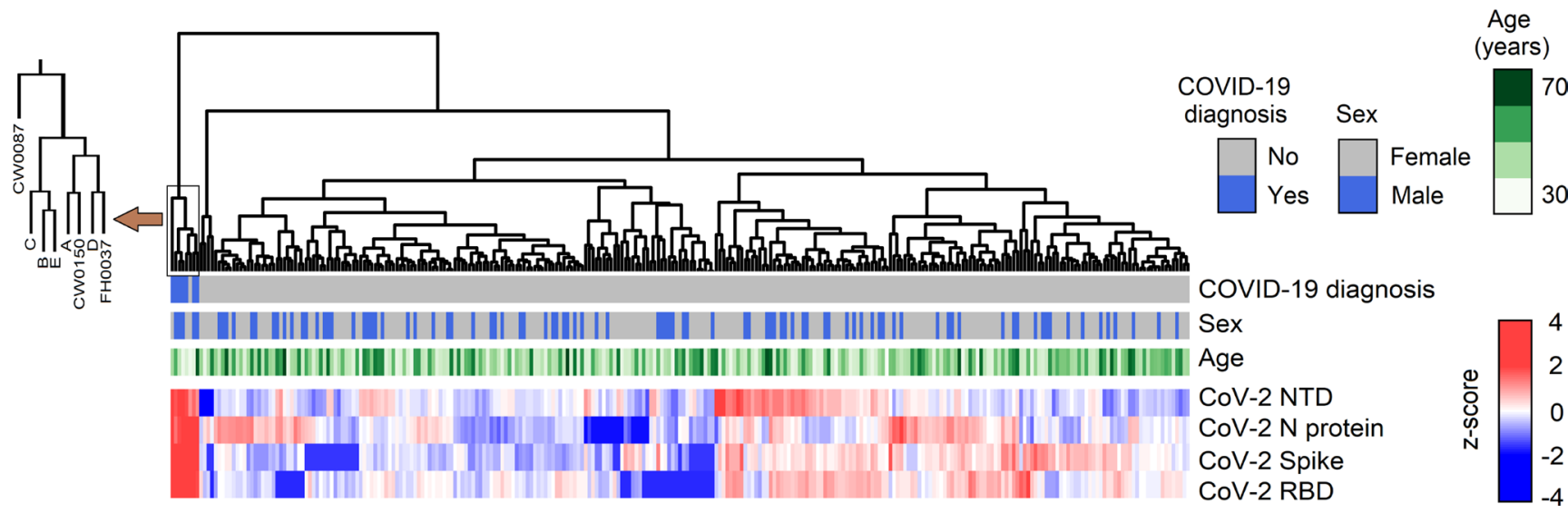

Figure 1. Hierarchical clustering of individual based on serum SARS-CoV-2 antibody reactivity profiles. COVID-19 diagnosis identifies convalescing individuals who had a positive viral test by PCR. This figure combines data from 276 study participants plus the 5 COVID-19 convalescent control sera. Color scale represents antibody reactivity as a $Z$ score.

individuals was due to nonspecific binding in the multiplex assay and to assess whether this antibody reactivity may represent cross-reactive antibody responses to circulating coronaviruses. To these ends, we determined whether the antibody reactivity against antigens in the multiplex assay could be outcompeted using either a cocktail of free SARS-CoV-2 RBD and full-length spike proteins - or the spike proteins from all 4 other circulating coronavirus spike proteins (OC43, HKU1, NL63, and 229E) pooled (Figure 2). Antibody reactivity was measured on serial dilutions from selected COVID-19 convalescent and uninfected sera selected on the basis of a high reactivity to full-length SARS-CoV-2 spike protein, its RBD, or its low reactivity to both of these antigens. As expected, the high SARS-CoV-2 spike and RBD antibody reactivity in COVID-19 convalescent sera was efficiently outcompeted by free SARS-CoV-2 spike and RBD proteins, but not by other free circulating coronavirus spike proteins (Figure 2, A and B).

Moreover, antibody reactivity to SARS-CoV-2 spike and RBD was partially outcompeted by circulating coronavirus spikes in uninfected individuals - this latter finding supports the argument that at least some of this SARS-CoV-2 antibody reactivity represented cross-reactivity toward circulating coronaviruses. Conversely, reactivity to circulating coronavirus spike proteins was efficiently outcompeted by spike protein from circulating coronaviruses but not by SARS-CoV-2 spike and RBD proteins (Figure 2, C and D). Therefore, this experiment confirms that SARS-CoV-2 spike and RBD antibody reactivity in uninfected individuals is saturatable, essentially excluding nonspecific binding in the multiplex assay. Interestingly, competition of SARS-CoV-2 spike antibody reactivity was higher in uninfected individuals with higher detectable SARS-CoV-2 spike or RBD antibody reactivity compared with individuals who showed low reactivity against these 2 antigens (Figure 2E). Unexpectedly, antibody reactivity against SARS-CoV-2 RBD in uninfected individuals was not efficiently competed by a fixed amount of SARS-CoV-2 RBD.

Seroreactivity thresholds defined in sera from immunologically naive infants. To unequivocally distinguish uninfected individuals who could have SARS-CoV-2 antibody reactivity, we defined the background of antibody reactivity of sera in the multiplex assay. We reasoned that infants would be immunologically naive, with the exception of maternal antibodies that are expected to wane gradually after birth; thus, their sera can be used to define antibody reactivity thresholds in uninfected adults in the multiplex assay. Using this assay, we measured antibody reactivity of sera from 45 infants less than 6 months of age and repeated this measurement in the same infants approximately 8 months later, after BC's lockdown period (Figure 3); the study included 21 infants in whom the first sera were obtained before the pandemic (i.e., before January 2020). In infant sera, reactivity to circulating coronaviruses was uniformly detected in the first set of blood samples, albeit at much lower levels than adults. In the second infant sample sets taken after July 1, 2020, antibody recognition of circulating coronaviruses had decreased to approximately 1000-fold lower levels compared with adults, consistent with a waning of maternal antibodies (Supplemental Figure 5). When comparing antibody reactivity of SARS-CoV-2 in the second postnatal infant sera, levels were up to 100-fold higher in uninfected adults compared with infants, regarding the different SARS-CoV-2 antigens (Figure 3). 
A

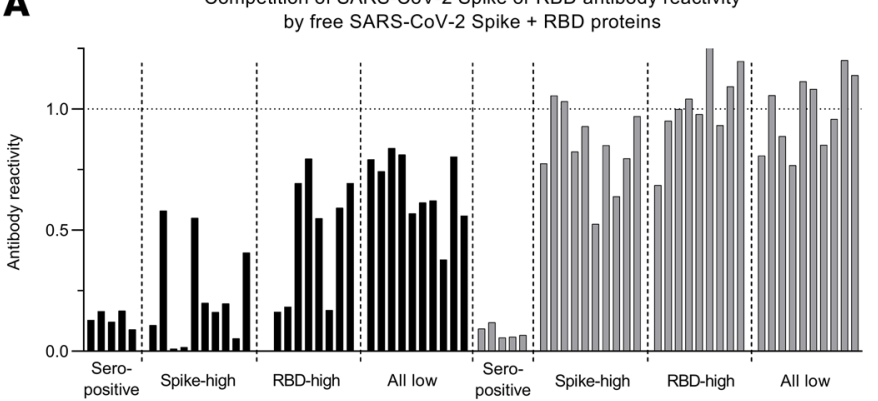

B Competition of SARS-CoV-2 Spike or RBD antibody reactivity by free Spike proteins from all four circulating coronaviruses

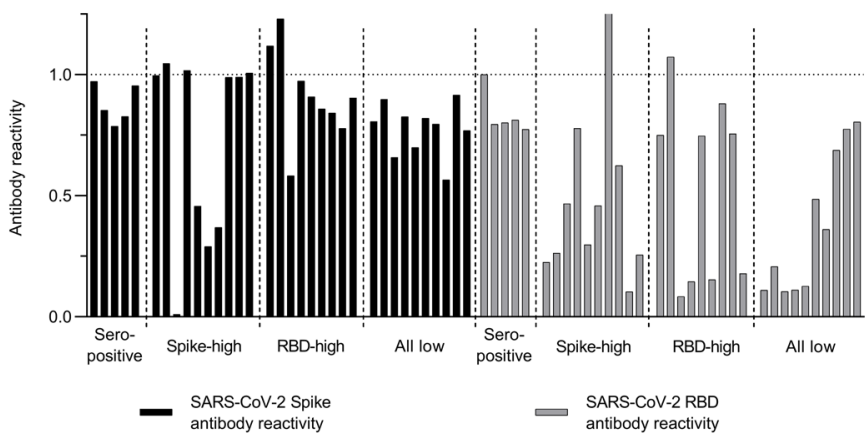

E Competition of SARS-CoV-2

Spike antibody reactivity

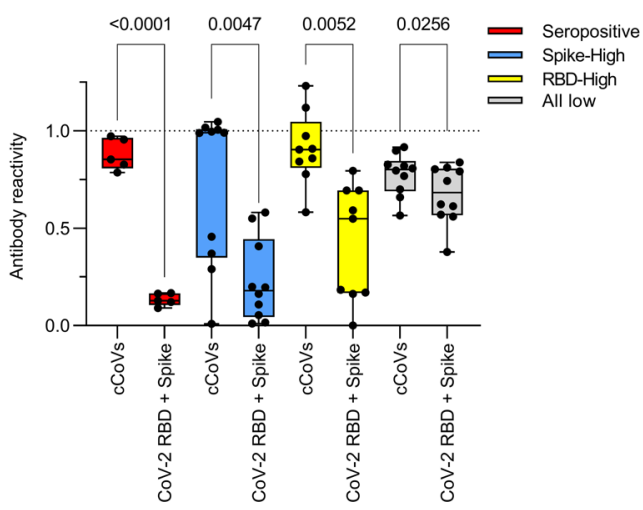

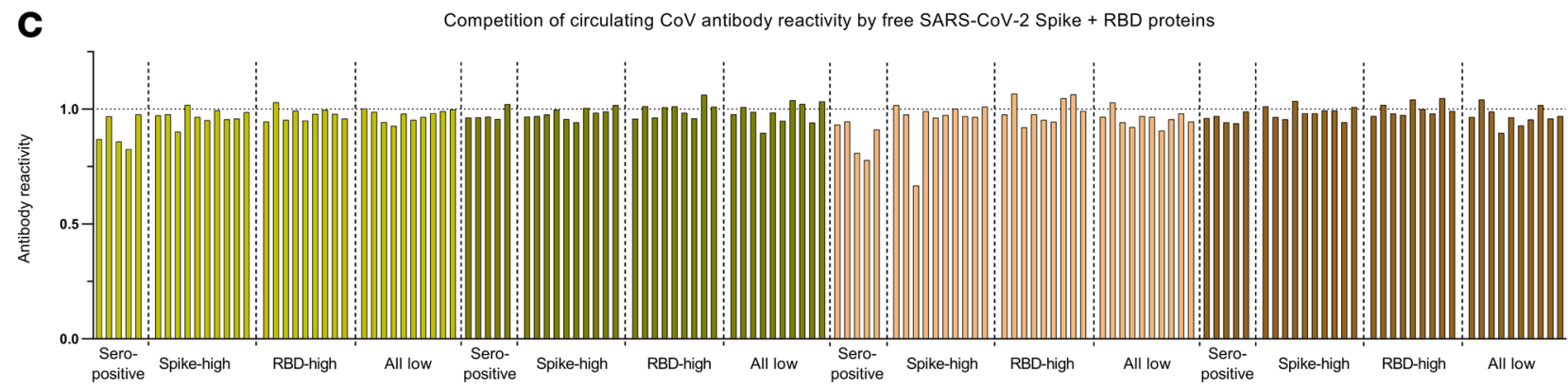

D

Competition of circulating coronavirus antibody reactivity by free Spike protein from all four circulating coronaviruses

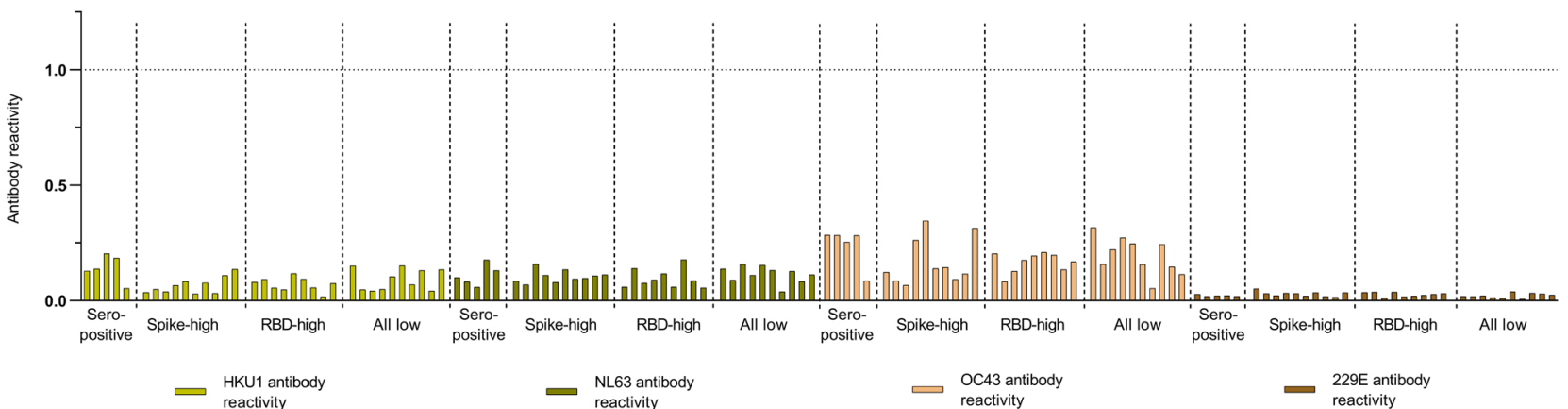

Figure 2. Specificity of SARS-CoV-2 antibody reactivity. (A and B) Competition of SARS-CoV-2 spike and RBD antibody reactivity by SARS-CoV-2 spike and RBD proteins (A) or by circulating coronaviruses (cCoVs) spike proteins (B). (C and D) Competition of cCoVs spike antibody reactivity by SARS-CoV-2 spike and RBD proteins (C) or cCoVs spike proteins (D). (E) Competition of SARS-CoV-2 spike antibody reactivity by SARS-CoV-2 spike and RBD proteins or cCoVs spike proteins, in COVID-19 convalescent sera (seropositive, $n=5$ ), or sera from uninfected individuals who showed highest SARS-CoV-2 spike ( $n$ $=10$ ) and high RBD $(n=9)$, or lowest SARS-CoV-2 spike and RBD antibody reactivity (all low; $n=10$ ). All values represent the ratios of antibody reactivity in competed samples over the antibody reactivity measured in absence of competing proteins (dash line). One sample in the RBD-high group failed, and these data are not shown. In E, data are represented as boxes (25th to 75 th percentile, line at median) and whiskers (minimum to maximum); comparisons were made using 2-tailed paired $t$ tests. 
Thus, these second infant samples allowed us to define effective thresholds for SARS-CoV-2 antibody reactivity in uninfected adults (Figure 3). Based on infants' sera, we estimate that between $90 \%$ and $99 \%$ of adults show positive antibody reactivity for SARS-CoV-2 spike, RBD, or the $\mathrm{N}$ antigen. Prepandemic sera showed similar antibody reactivity, therefore excluding the possibility that the reactivity in adults after the first pandemic wave is due to undiagnosed exposures to the virus in the study population. This baseline, preexisting SARS-CoV-2 cross-reactivity in uninfected adults was evenly distributed according to age, sex, travel history, or whether participants were healthcare workers (HCW), and the data were independent of participants' reporting "COVID-19-like" symptoms (Supplemental Figure 6).

Further characterization of SARS-CoV-2 antibody reactivity in uninfected adults. To map this antibody reactivity on the viral proteome, we used a SPOT array assay where peptides broadly covering the SARS$\mathrm{CoV}-2$ proteome were directly synthesized on a cellulose membrane (Supplemental Figure 7). To enrich for high-affinity antibodies, sera from individuals who showed high spike or RBD antibody reactivity were compared with infant samples. As shown in Figure 4, we detected high antibody reactivity against nonstructural proteins, particularly the nonstructural protein 2 (nsp2) and nsp15 encoded in the replicase polypeptides ORF1a and ORF1b. RBD-high samples showed the strongest antibody reactivity encompassing RBD, as well as the S1 and the S2 peptides, indicating a diverse anti-SARS-CoV-2 antibody reactivity linked to a high RBD antibody cross-reactivity. This cross-reactivity was also detected in randomly selected prepandemic sera, which demonstrated preexisting recognition prior to the SARS-CoV-2 pandemic. Importantly, we detected no antibody reactivity against any viral peptides in infants' sera.

\section{Discussion}

In this study, we estimated that $0.60 \%(95 \% \mathrm{CI}, 0 \%-2.71 \%)$ of the study population showed clear evidence of a prior infection with SARS-CoV-2. The combination of a highly specific commercial CLIA assay and a highly sensitive multiplex assay allowed us to distinguish individuals who have been infected with SARSCoV-2 from those who have not. This prevalence of SARS-CoV-2 infections was identical to the $0.55 \%$ prevalence reported by the $\mathrm{BC}$ CDC on 885 residual sera obtained from an outpatient laboratory network in the Lower Mainland of BC between May 15 and May 27, 2020. Data from the BC CDC represent a wider geographical catchment and do not specifically target HCW (12). The current study confirms that COVID-19 transmission in BC after the first wave was low, even among HCW, contrasting with a high seroprevalence reported among HCW in other studies (13-15), which may be attributed to the very low number of total tested cases in $\mathrm{BC}$ during the first wave.

The main finding in this study is that, at a population level, the vast majority of adults show antibody reactivity against SARS-CoV-2 antigens. BC reported its first COVID-19 case on January 29, 2020, with the first documented case of community transmission on March 5, 2020. The first pandemic wave peaked between the third week of March and late April 2020 (11). As of May 17, only 2445 diagnosed COVID-19 cases (approximately 49 of 100,000 population) had been reported in BC after the first wave, which was the lowest rate in Canada and one of the lowest rates in North America. Because of a relatively low number of COVID-19 cases in BC after the first wave, it is extremely unlikely that this antibody reactivity results from a direct exposure to SARS-CoV-2. Moreover, findings of similar antibody reactivity in prepandemic adult sera and from sera obtained from infants younger than 1 year of age confirms that we are detecting genuine cross-reactivity rather than reactivity to SARS-CoV-2 from asymptomatic COVID-19 cases.

Our findings are consistent with another study in which prepandemic sera exhibited cross-reactive IgG antibody reactivity with conserved epitopes in SARS-CoV-2 proteins (S2 and N) (5). The higher prevalence of preexisting antibody reactivity in uninfected adults in our cohort compared with this previous study may be explained by the high sensitivity of our assay and evidence of positive seroreactivity in those individuals informed by the infant sera. However, whereas these previous studies have quantified cross-reactivity in selected sera, to the best of our knowledge, the current study is the first to determine SARS-CoV-2 antibody reactivity at the population level. The fact that we measured antibody reactivity between infected and uninfected individuals in the same population and time period in the current study also eliminates recruitment or sampling biases and is another major strength of this study.

The presence of preexisting SARS-CoV-2 antibody reactivity in uninfected individuals in the current study is consistent with the detection of T cell reactivity against SARS-CoV-2 in about $40 \%$ of uninfected individuals $(3,4)$. This raises an important question: what is the antigenic source of this antibody reactivity? 


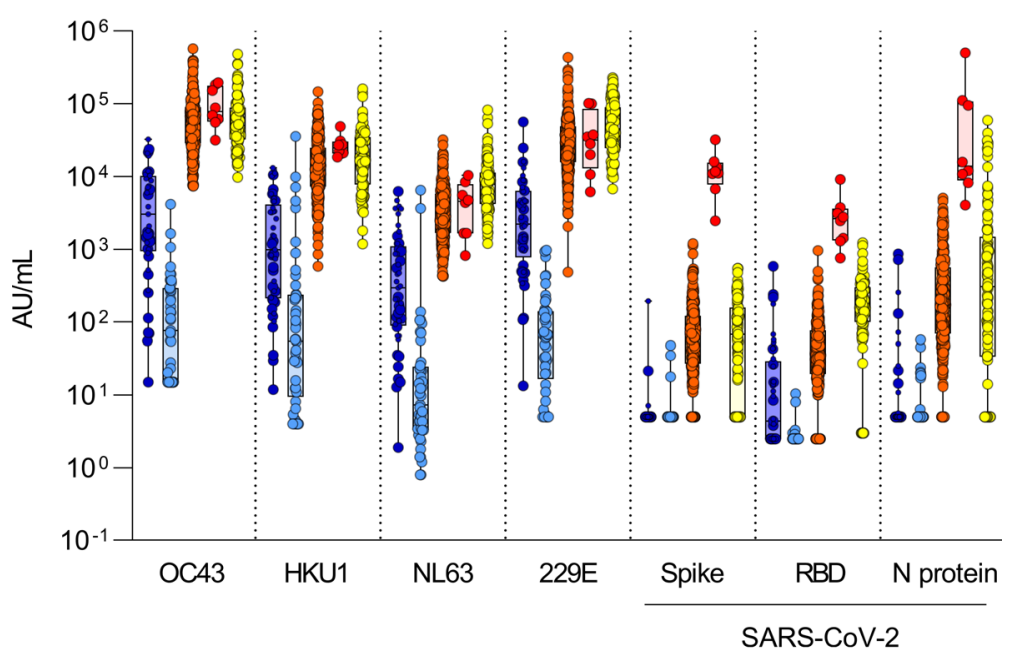

Figure 3. Thresholds of antibody reactivity based on infants' sera. Comparison of antibody reactivity (AU/mL) in infants sampled before 6 months of age (darker blue) and again about 8 months later (lighter blue; $n=45$ ), in SARSCoV-2-uninfected (orange; $n=273$ ), in SARS-CoV-2-infected (convalescent) adults (red; $n=8$ ), and in prepandemic sera (yellow; $n$ =99). Infants sampled before the pandemic (January 1, 2020) are represented by the larger circle symbols, whereas infants sampled after January 1, 2020, are shown using the small circle symbols. Boxes represent median with 25th and 75th percentiles with positive/negative antibody reactivity thresholds for SARS-CoV-2 spike calculated at the 99th percentile for value distribution (10.00 AU/mL), RBD (10.00 AU/mL), and $\mathrm{N}$ protein $(10.00 \mathrm{AU} /$

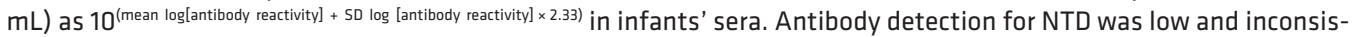
tent between experiments; therefore, the data are not presented and reactivity thresholds were not calculated.

Competition experiments and correlatives analyses indicate that it may, in part, be attributable to cross-reactivity against circulating coronaviruses. Most humans become infected with circulating coronaviruses by their second year of age (16). On the one hand, correlations between SARS-CoV-2 and antibody reactivity against either HKU1, N63L, or 229E, but not OC43, could reflect seasonal variations in recent exposure to common coronaviruses $(10,17)$. On the other hand, the high antibody reactivity to SARS$\mathrm{CoV}$ in individuals in this study likely represents cross-reactivity due to the higher $(>75 \%)$ sequence similarity between SARS-CoV and SARS-CoV-2 $(18,19)$, rather than a previous exposure to SARS-CoV.

The data presented in this study shed light on another important question: what region of the virus does this preexisting antibody reactivity bind to? We found in our peptide mapping experiments that it is broadly distributed across the viral proteome, including whole spike, and proteins encoding the viral replication complex. The binding to ORF polypeptides could be a sign of infection by circulating coronaviruses that share conserved sequences with SARS-CoV-2. High antibody reactivity against nonstructural ORF proteins was reported in another study using a VirScan peptide mapping approach on prepandemic sera (6). However, due to a lower sensitivity of the assay, antibody reactivity against spike was not detected in the latter study. Here, we confirm that this preexisting antibody reactivity involves structural external elements of the virus in both epitope mapping and competition experiments.

It is unclear whether this antibody reactivity may confer clinical benefits - for instance, modulating the severity of a SARS-CoV-2 infection. Data indicate that a past circulating coronavirus infection may decrease the severity of a subsequent SARS-CoV-2 infection (20). Others have linked preexisting seroreactivity against circulating coronaviruses to increased SARS-CoV-2 pseudovirus neutralization in vitro (5), although this remains debated. Individuals with high RBD reactivity showed the most structurally diverse antibody reactivity against spike epitopes, which may enhance viral clearance in addition to the improved neutralizing activity specific to RBD-specific antibodies. Indeed, strong antibody response to RBD have been linked to improved clinical outcomes from COVID-19 (21). However, reactivity against RBD in the multiplex assay was not competed by soluble RBD in uninfected individuals, despite that this reactivity was almost completely abrogated in COVID-19 convalescent sera. The latter finding is consistent with another study that showed that preexisting antibody reactivity against SARS-CoV-2 in prepandemic sera could be efficiently competed by a soluble S1 (that contains the RBD domain) but not a soluble S2 subunit of the spike protein (5). Notably, we were also unable to detect ACE2 receptor binding inhibition from 


\begin{tabular}{|c|c|c|c|c|c|c|c|c|c|c|c|c|c|c|c|c|c|c|c|c|c|c|c|c|c|c|c|c|c|c|c|c|c|c|c|c|c|c|c|c|c|c|c|c|c|c|c|c|c|}
\hline \multirow{5}{*}{$\begin{array}{c}\begin{array}{c}\text { Protein and } \\
\text { Domain }\end{array} \\
\text { Spike S1-NTD }\end{array}$} & \multirow{5}{*}{$\begin{array}{c}\text { Amino Acid } \\
\text { Residue Span }\end{array}$} & \multicolumn{5}{|c|}{ C| $\begin{array}{c}\text { Pre-COVID- } \\
19\end{array}$} & & & & & & Spik & ke-l & hig & h s & sel & ect & ed & sar & Impl & oles & & & & & & & & Rec & cept & tor & bin & ndin & ng & & $\begin{array}{ll}\mathrm{ma} \\
\mathrm{amr}\end{array}$ & in ( & $\begin{array}{l}\text { RE } \\
s\end{array}$ & BD) & & & & & & & & & & \\
\hline & & & 1 & & & & 1 & & & & & & & & & & & & 1 & & & & 2 & & & & & 2 & & & & & 3 & 1 & & & 8 & & & & & & 1 & & & & & & \\
\hline & & & 11 & & & & & & & & & 1 & & & & & 1 & & 1 & 2 & 1 & & 1 & 2 & 1 & & & & 1 & & 1 & 1 & 1 & 1 & 1 & 2 & & 2 & & & 2 & 12 & 23 & 3 & 12 & & & & \\
\hline & & & & & & 2 & & & & & & & & 1 & & & 1 & 1 & 2 & & 1 & 1 & & 2 & 1 & 2 & & 1 & & 2 & & 2 & 1 & & 1 & 1 & 2 & 1 & 2 & 2 & 2 & 12 & 21 & & $\begin{array}{ll}3 & 2 \\
3\end{array}$ & & & 1 & \\
\hline & & & & 2 & & & & & & & & & & & & & & & & & & & & & 2 & & & & & & 1 & & & & 1 & & & & & & 1 & 1 & & & & & & & \\
\hline Snike S1-RBD & $315-329$ & & & & & & & & & & & & & & & & & & & & & & & & & & 1 & & & & 1 & & 1 & 1 & 2 & 1 & 1 & 1 & & 1 & 2 & 22 & & 21 & & & & & \\
\hline 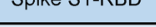 & $322-336$ & & & & 1 & & & & & & & & & 1 & & & & & 2 & & 1 & & 1 & 2 & 1 & 2 & 1 & 2 & & 3 & & 1 & & & & 4 & 2 & 3 & 1 & 2 & 5 & $3: 2$ & \begin{tabular}{l|l}
3 & 3 \\
\end{tabular} & & \begin{tabular}{l|l}
3 & 4 \\
\end{tabular} & & & & \\
\hline Snike S1-RBM & $463-477$ & & & & & 1 & & & & & & & & 1 & & & & 2 & 1 & & 1 & 2 & 1 & 1 & 2 & 3 & & 1 & & & & 1 & 1 & & & 2 & & 1 & 1 & 1 & 1 & 1 & 11 & 13 & & & & & \\
\hline spIKe si-RDIVI & $491-505$ & & & & & & & & & & & & & 1 & & & 1 & & & & 1 & 1 & & & 2 & 1 & 1 & 1 & & 1 & & 1 & & & & 2 & 2 & 1 & & & 1 & 11 & 1 & 2 & 22 & & & & \\
\hline & $551-565$ & & & & 1 & & & & 1 & 1 & 1 & & & & & & 1 & & & 1 & & & 1 & & & 1 & 1 & & 1 & & 1 & 1 & 3 & 3 & 1 & 1 & 2 & 2 & 8 & & 1 & 1 & 1 & 13 & $\begin{array}{ll}32 \\
3\end{array}$ & & & & 1 \\
\hline Spike S1-CTD1 & $558-572$ & & & & & 1 & & & & & & & & & & & & & & & & & & & 1 & & & & 1 & & & & & & 1 & & & & & & & & 1 & & & & & & \\
\hline & $571-585$ & & & & & & & & & & & & & & & & & & & & & & & & & & & & & & & & & & & & & & & & & & 2 & & & & & & \\
\hline Spike S1-CTD2 & $625-640$ & & & 6 & 2 & & & & & 2 & 1 & 2 & 1 & 1 & & 1 & & & 1 & & 1 & & & & 3 & 1 & 1 & & & & 1 & 2 & 1 & 1 & 2 & 1 & & 1 & 1 & & 2 & 2 & 12 & $2 \sqrt{2}$ & & & & & \\
\hline & $713-727$ & & $\begin{array}{ll}21 \\
\end{array}$ & & & 1 & & & & & & & & & & & & & & & & 2 & & & & & 2 & & & & 1 & & 1 & 1 & 1 & 1 & & & & 2 & & & 12 & 2 & 1 & & & & \\
\hline Snike S? & $766-780$ & & & 2 & & 1 & & & & & & 1 & & & & & 4 & & & & & & & & & & & & & & & & & & & & & & & & & 2 & & & 22 & & & & \\
\hline spike š & $791-805$ & & 1 & 1 & & 3 & & & & & & & & & & & & & & & & 1 & & & & & 1 & 1 & 2 & & 1 & & 3 & 2 & 1 & 2 & 1 & & 5 & & 1 & 22 & 22 & 2 & 23 & & & & \\
\hline & $795-807$ & & 1 & & & & & & & & 3 & & & & & 1 & & & & & & & & & 1 & & & & 4 & & & & & 1 & 2 & & 2 & & 1 & 4 & & & 12 & 2 & 2 & & & & \\
\hline Spike S2-FP & $810-824$ & & 2 & 5 & 1 & 2 & 1 & & & & & & 2 & & 1 & 1 & & & & & 2 & & 3 & 1 & & & 2 & 1 & & 2 & 3 & & 1 & 1 & 3 & 2 & 1 & 3 & 1 & $3 \mid$ & 6 & 12 & \begin{tabular}{l|l}
23 \\
\end{tabular} & 3 & 23 & & & & 1 \\
\hline Spike S2 & $879-893$ & & 2 & & 4 & 3 & & & & & & & & & & & & & & & & & & & & & & & & & 2 & & 2 & 1 & 1 & 1 & 1 & 1 & 1 & 2 & 3 & 1 & 12 & 2 & - & & & & \\
\hline Spike S2-HR1 & $927-941$ & & & & 1 & & & & 3 & 1 & & & & & & & & & & & & & & & & & & 1 & & & & & & - & - & - & 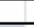 & & & & & 2 & & & 3 & & & & \\
\hline $\mathrm{S} 2-\mathrm{HR} 1 / \mathrm{CH}$ & $975-989$ & & & & 2 & & & & & & & & & & & 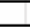 & & & & & & & & & & 3 & & & & & 2 & & & & & & 1 & & & 2 & & & 1 & 1 & & & & & \\
\hline & $987-1001$ & & & & 2 & 1 & & & & & & & & & & & & & & & & & & & & 2 & & & & 2 & & 1 & & & & & 1 & 1 & & 1 & 1 & 2 & & & 22 & & & & \\
\hline$S<-C H$ & $989-1003$ & & & & 1 & & & & & & & & & & & & & & & & & & & & & 1 & & & & & & & & 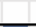 & 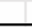 & & & 1 & & & & 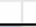 & & 2 & 21 & & & & \\
\hline S2-CR & $1143-1157$ & & & & & & & & & & & & & & & & & & 1 & & & & & & & & & & & 1 & & & & 3 & & & 1 & & & & & 1 & & & 12 & & & & \\
\hline $8<-C D$ & $1147-1161$ & & & & & & & & & & & & & & 1 & 1 & & & & 4 & & & & & & 1 & & & 1 & & 1 & & & 4 & 2 & & & & 1 & & & & & & 1 & & & & \\
\hline & $13-27$ & & & & & & & & & & & & & & & & & & & & & & & & & & & & & & & & & & 1 & & 1 & & 1 & 1 & 1 & 2 & & & 1 & & & & \\
\hline & $156-170$ & & & & & & & & & & & & & & 3 & & & & & & & & & & & & & & & & & & & & 4 & & & & & & & & & & & & & & \\
\hline Nucleocapsid N & $241-255$ & & & & & & & & & & & & & & & & & & & & 2 & 1 & & & 1 & & & & 2 & & & & & 1 & & & 1 & & & & 1 & & & & & & & & \\
\hline & $271-285$ & & & & & & & & & & & & & & & & & & & & & & & 2 & & & & & & & & & & & & & & & & & & & & 1 & & & & & \\
\hline & $389-403$ & & & & & & & & & & & & & & & & & & & & & & & & & & & & & & & & & & & & & & & & & 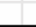 & & 2 & & & & & \\
\hline Membrane M & $3-17$ & & 1 & & & & & & & & & & & & & & & & & & & & 1 & & & & & & & & & & & & & & & & 1 & & & 1 & & & & & & & \\
\hline Iviemorane iv & $7-23$ & & 2 & & & 1 & & & & & & & & & & & & & & & & & & & & & & & & & & & & 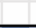 & & & & 1 & & & & 11 & 11 & & & & & & \\
\hline ORF1ansp2 & $154-168$ & & 14 & 1 & 1 & 2 & 1 & 1 & 1 & 2 & 2 & 2 & 2 & & 3 & 1 & 2 & 3 & 2 & 3 & 2 & |3| & 3 & 2 & 3 & 3 & 2 & 3 & 2 & 3 & 2 & 3 & 2 & 3 & 2 & 4 & 3 & 3 & 3 & 5 & 3 & 42 & & & 44 & & & & \\
\hline URr lanspe & $735-749$ & & & & & 1 & & & & & & & 1 & & & & & 1 & 1 & 1 & 1 & 1 & 2 & 2 & 2 & 2 & & 1 & & 1 & & 3 & & & & 2 & & 2 & 1 & 4 & 2 & 22 & & 32 & 21 & & & & \\
\hline ORF1a nsp3 & $876-890$ & & & & & & & & & & & & & & & & 1 & & & & & & & 9 & & & & & & & & & & 3 & & 3 & & - & & & 1 & & 4 & & & & & & \\
\hline ORF1b nsp8/9 & $4133-4147$ & & & & & & & & & & & & & & & 5 & & & & & & & & & & 2 & & & 1 & & & & & & & & & 1 & & 1 & & & & & & & & & \\
\hline 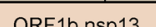 & $5447-5461$ & & & & 2 & 2 & & & & & & & & & & & & 4 & & & & & 1 & & & & & & & & & 3 & & & & & & & 4 & & 2 & 2 & 1 & 4 & 1 & & & & \\
\hline Uretioniso & $5605-5619$ & & & & 2 & & & 3 & & & & & & & & & & & & & & & & & & & & & & & & & & & 1 & & & 1 & & & & 1 & & & 1 & & & & \\
\hline ORF1b nsp14 & $6053-6067$ & & 2 & & & 1 & & & & & & & & & & & & & & & & & & & 1 & 1 & & & & & 1 & & & & & & & & 1 & 1 & & 1 & 11 & 11 & 11 & & & & \\
\hline ORF1b nsp15 & $6590-6604$ & & & & & & & & & & & & & & & & & & & & & & & & & & & & & & & & & & & & & & & & & & & & & & & & \\
\hline & $6722-6736$ & & & & 1 & & & & & & & 2 & 2 & 3 & 2 & 1 & 2 & 3 & 1 & 3 & 2 & 3 & 3 & 2 & 2 & 3 & 1 & 2 & 1 & 2 & 1 & 3 & 3 & & 2 & 3 & 1 & 5 & 2 & 5 & 2 & $3 \sqrt{2}$ & \begin{tabular}{l|l}
4 & 3 \\
\end{tabular} & & 41 & & & & \\
\hline ORF3a & $31-45$ & & & & & & & & & & & & & & & & & & 1 & & & & & & & & & & 1 & & 3 & 1 & 2 & & 2 & & & 1 & 1 & & 1 & & \begin{tabular}{l|l}
3 & 2 \\
\end{tabular} & 21 & 1 & & & & \\
\hline
\end{tabular}

Figure 4. Mapping of SARS-CoV-2 antibody reactivity in sera from uninfected individuals. Serum antibody binding to 15-mer peptides distributed across the SARS-CoV-2 proteome or an IgG-binding peptide (positive control), from 5 randomly selected prepandemic samples, adults showing high level of spike or RBD reactivity ( $n=20$ each), or infants $(n=5)$. Values represent signals on a scale from 0 to 10 , after subtracting background. The column labeled C shows the immunoreactivity signal in the absence of sera, but with the addition of anti-human IgA, IgM, and IgC horse-radish peroxidase-coupled secondary antibody. In the spike protein, labels indicate the N-terminal (NTD), C-terminal (CTD), or receptor-binding (RBD) domains, receptor-binding motif (RBM), heptad repeat sequence (HR1), central helix (CH), or connector domain (CD); N, nucleocapsid protein; M, membrane protein; ORF, open-reading frame polypeptide proteins; nsp, nonstructural proteins.

sera of uninfected individuals (not shown), which could indicate that the preexisting antibody reactivity against SARS-CoV-2 in uninfected adults represents an excess of low-affinity antibodies that have poor overall viral neutralizing potential. This may not be surprising given that viral neutralization improves generally with affinity maturation, an antigen-driven process that requires cognate interaction by B cells, in collaboration with follicular T cells. Similarly, preexisting, highly variable low-avidivity SARS-CoV-2 CD4 memory $\mathrm{T}$ cells cross-reactive to circulating coronaviruses appeared less protective in uninfected adults (22). More studies are needed to understand the origin of preexisting SARS-CoV-2 antibodies and their impact on COVID-19 severity.

In conclusion, this study reveals common preexisting, broadly reactive SARS-CoV-2 antibodies in uninfected adults. These findings warrant larger studies to understand how these antibodies affect the severity of COVID-19, as well as the quality and longevity of responses to SARS-CoV-2 vaccines.

\section{Methods}

Study design. Prospective cross-sectional study after the first pandemic wave in BC.

Participants. Adults over 18 years of age from the greater Vancouver metropolitan area were included if they did not have active COVID-19, did not require self-isolation as per BC provincial public measures, or had recovered from COVID-19 at least 14 days prior to the study visit and blood collection. Blood was drawn in gold-top serum separator tubes with polymer gel (BD Biosciences, catalog 367989); after at least 30 minutes of clotting at room temperature, the blood sample was then centrifuged at $1400 \mathrm{~g}$ for 10 minutes at room temperature to obtain serum aliquots that were frozen at $-80^{\circ} \mathrm{C}$ within 4 hours of collection. Adult prepandemic sera were all obtained before January 1, 2020. Infants' sera were collected before discharge from hospital at birth (first sample) and after June 11, 2020 (second sample), as part of a study examining antibody responses to respiratory viruses. 
Recruitment. Greater Vancouver is the main urban center in BC and the third largest metropolitan area in Canada, with a population of 2.5 million. Study participants were invited by an email sent to clinical departments of the BC Children's \& Women's (C\&W) Hospitals (the largest pediatric referral center in BC, located in Vancouver, and where no cases of COVID-19 were admitted during the pandemic's first wave) and its affiliated BC Children's Research Institute (BCCHR). The study was also advertised to hospitalists, anesthesiologists, and critical care physicians at SMH (located approximately $27 \mathrm{~km}$ from Vancouver). To minimize recruitment bias, all adults who responded to the invitation email and returned their signed consent form were enrolled sequentially and invited to give a blood sample, without triaging. Blood samples were collected between May 17 and June 19, 2020.

Study size. Since there were little population seroprevalence data available at the time and none in BC or Canada, no a priori sample size calculation was performed. The recruitment period was, therefore, defined by convenience over a 3-week period of enrollment, in order to obtain baseline data.

Multiplex antibody assay. A highly sensitive multiplex (10-plex) assay (Meso Scale Diagnostics, catalog $\mathrm{K} 15369 \mathrm{U})$ where each antigen is "spotted" into a single well of a 96-well plate (23) was used to measure antibody profiles against 4 SARS-CoV-2 antigens: the trimeric (whole) S-2P native spike protein, its RBD, its NTD (24), and N protein; the trimeric SARS-CoV spike protein; and spike proteins from circulating $\beta$-coronaviruses (HKU1, OC43) and $\alpha$-coronaviruses (229E, NL63), plus BSA, a negative control. Briefly, after blocking wells with 5\% BSA, sera were added at 4 dilutions $(1: 100,1: 800,1: 3200$, and 1:10,000) and incubated with shaking for 2 hours. Sulfo-tag-labeled anti-IgG detection antibodies were added, and the electrochemiluminescence signal was read using the MSD Sector 600 instrument (Meso Scale Diagnostics). Initial AUC of the electrochemiluminescence values for antibody detection were well above the BSA background for all sera for SARS-CoV-2 antigens, except for 1 sera for the RBD and N antigens and 10 sera for the NTD antigen. Samples were rescreened again in a second set of experiments after Meso Scale Diagnostics provided standards. Results are presented as dilution-corrected interpolated values from a standard curve with assigned AU/mL. Assignment of AU/mL of serum was performed by Meso Scale Diagnostics and is designed such that values are comparable with an International Standard Serum (ISS), so that bridging to a WHO International Standard will be possible in the future.

Competition experiments. Eight 2-fold dilutions of sera prediluted in a ratio of 1:50 assay diluent were added to an equal volume of assay diluent (control) or to assay diluent mixes containing $5 \mu \mathrm{g} / \mathrm{mL}$ SARS-CoV-2 spike and $5 \mu \mathrm{g} / \mathrm{mL}$ RBD proteins (SARS-CoV-2 RBD-spike cocktail), or $5 \mu \mathrm{g} / \mathrm{mL}$ spike proteins from all 4 circulating coronaviruses (HKU1, OC43, 229E, NL63; circulating coronaviruses [cCoVs] cocktail) (25), for an on-plate assay dilution of 1:100 through 1:12,800. The dilution series was incubated for 30 minutes at room temperature and then analyzed using the multiplex antibody assay protocol, as mentioned previously.

CLIA antibody assay. Total antibody (IgA, IgG, and $\operatorname{IgM})$ against recombinant spike (S1) protein was determined using the VITROS 5600 analyzer (Ortho-Clinical Diagnostics) according to manufacturer instructions. This is a Health Canada and FDA-licensed qualitative assay with reported performance and in-house validation indicating sensitivities $>7$ days after onset range between $96 \%$ and $100 \%$, and specificities from $99 \%$ to $100 \%(26,27)$.

SPOT peptide array. Forty-one 15-mer peptides selected based on their reactivity on convalescent samples and immunogenicity, and that were distributed over the entire SARS-CoV-2 proteome, were synthesized on a cellulose trioxatridecanediamine membrane using a MultiPep synthesizer (CEM) (28). Additionally, each membrane contained a human-IgG binding peptide as positive control (29). These in-house-made membranes were incubated with a 1:400 dilution of sera and incubated for 2 hours at room temperature. A copy of the array was also incubated with Tris buffered saline with Tween 20 only, as a negative control. After washing, membranes were incubated with secondary antibody (HRP-conjugated goat anti-human IgA + IgG + IgM polyclonal antibody, Jackson ImmunoResearch Inc., catalog 109-035-064) at a 1:30,000 dilution for another 2 hours, and detection was carried out using enhanced chemiluminescence detection, with 8 images captured over an exposure time of 50 seconds. The grayscale of images represented as numeric values between 0 and 10 were used before applying a uniform background correction of 1 .

Variables. The following information was collected from participants by questionnaire: age; sex; the first 3 digits of their postal code; HCW status (and whether they worked at C\&W or SMH); history of travel outside BC since January 1, 2020; and history of COVID-19 symptoms and testing. SARS-CoV-2-exposed cases were defined by a positive result on the commercial CLIA assay, validated for sensitivity by antibody profiling on the multiplex assay. 
Statistics. The seroprevalence from a SARS-CoV-2 exposure was adjusted for bias due to false-positive and false-negative tests using the Greenland method (30). Differences in proportions were calculated using a Fisher's exact test, with significance threshold at $P<0.05$. Hierarchical clustering of antibody levels (based on the multiplex assay) was performed on log-transformed, $Z$ score-normalized serology data, using the complete linkage agglomeration method and Euclidean distance measures. Spearman correlations between antibody levels and metavariables were adjusted for multiple testing using the Benjamini-Hochberg FDR $=0.05$. There were no missing data. For competition experiments, same-sample groups were compared using 2-tailed paired $t$ tests. Analyses were conducted in $\mathrm{R}$ version 4.0.2, $\mathrm{R}$ Studio version 3.6.2, and GraphPad Prism version 8.4.

Study approval. Written informed consent was obtained from all participants. The study procedures were approved by the University of British Columbia (UBC) C\&W Research Ethic Board (H20-01205; H18-01724).

\section{Author contributions}

AM, CM, and SD coordinated the study sample accrual and blood processing in Vancouver. JG and DM coordinated recruitment at SMH. CC collated the data and helped with data analysis. SEO performed the multiplex assay, with help from SN and MB. MG provided important input into the study design. $\mathrm{JM}$ and DE expressed and provided the soluble proteins for the multiplex assays and competition experiments. ACL, IS, and ANJ revised the manuscript. MI supervised the statistical analyses. VEB and DMG supervised the commercial CLIA testing of samples. DFHW and SP performed the SPOT peptide array analysis. PML and ABM supervised the study in Vancouver and at the NAID/NIH, respectively. AM, CM, SD, DCD, and PML wrote the manuscript's first draft. All authors contributed to the study design, data analysis, and reviewing the manuscript, and they accept the article submission in its final form. The order of co-first authors was determined based on their earlier involvement at the study design stage.

\section{Acknowledgments}

We thank Lauren Muttucomaroe, Esther Alonso-Prieto, and Lisa-Marie Candeias for help with recruitment; Linda Warner and Stuart Turvey for lending essential staff resources; Kim Schmidt and Kiara Gibbons for advertising the study; and study participants who have generously donated their time and blood samples. AM is supported by a Mining for Miracles postdoctoral award from the British Columbia Children's Hospital (BCCH) Foundation. CC is supported by BCCHRI Research Institute and UBC Faculty of Medicine Summer Student Research Program Studentships. AL is supported by a UBC Four Year Fellowship. PML holds a salary award from the BCCH Foundation through the Investigator Grant Award Program. ANJ and MG acknowledge funding from the Michael Smith Foundation for Health Research. This study was funded by the BC Children's Hospital Foundation (to PML), the Intramural Research Program of the Vaccine Research Centre (VRC) at the National Institute of Allergy and Infectious Diseases (NIAID), NIH (to ABM and DCD). These funders did not play a role in the design, planning, execution, analysis, or publication of the study. The study was also funded in part by the Government of Canada via its COVID-19 Immunity Task Force.

Address correspondence to: Pascal M. Lavoie, BC Children's Hospital Research Institute, 4th Floor, Translational Research Building, 950 West 28th Avenue, Vancouver, British Columbia V5Z 4H4, Canada. Phone: 604.875.2135; Email: plavoie@bcchr.ca.

1. Long QX, et al. Antibody responses to SARS-CoV-2 in patients with COVID-19. Nat Med. 2020;26(6):845-848.

2. To KK, et al. Temporal profiles of viral load in posterior oropharyngeal saliva samples and serum antibody responses during infection by SARS-CoV-2: an observational cohort study. Lancet Infect Dis. 2020;20(5):565-574.

3. Mateus J, et al. Selective and cross-reactive SARS-CoV-2 T cell epitopes in unexposed humans. Science. 2020;370(6512):89-94.

4. Braun J, et al. SARS-CoV-2-reactive T cells in healthy donors and patients with COVID-19. Nature. 2020;587(7833):270-274.

5. Ng KW, et al. Preexisting and de novo humoral immunity to SARS-CoV-2 in humans. Science. 2020;370(6522):1339-1343.

6. Shrock E, et al. Viral epitope profiling of COVID-19 patients reveals cross-reactivity and correlates of severity. Science. 2020;370(6520):eabd4250.

7. National SARS-CoV-2 Serology Assay Evaluation Group. Performance characteristics of five immunoassays for SARS-CoV-2: a head-to-head benchmark comparison. Lancet Infect Dis. 2020;20(12):1390-1400.

8. Su S, et al. Epidemiology, genetic recombination, and pathogenesis of Coronaviruses. Trends Microbiol. 2016;24(6):490-502.

9. Hicks J, et al. Serologic cross-reactivity of SARS-CoV-2 with endemic and seasonal Betacoronaviruses [preprint]. https://doi. org/10.1101/2020.06.22.20137695. Posted on medRxiv June 23, 2020.

10. Monto AS, et al. Coronavirus occurrence and transmission over 8 years in the HIVE cohort of households in Michigan. J Infect 
Dis. 2020;222(1):9-16.

11. Callow KA, et al. The time course of the immune response to experimental coronavirus infection of man. Epidemiol Infect. 1990;105(2):435-446.

12. Skowronski D, et al. Low SARS-CoV-2 sero-prevalence based on anonymized residual sero-survey before and after first wave measures in British Columbia, Canada, March-May 2020 [preprint]. https://doi.org/10.1101/2020.07.13.20153148. Posted on medRxiv July 15, 2020.

13. Stubblefield WB, et al. Seroprevalence of SARS-CoV-2 among frontline healthcare personnel during the first month of caring for COVID-19 patients - Nashville, Tennessee [published online July 6, 2020]. Clin Infect Dis https://doi.org/10.1093/cid/ciaa936

14. Houlihan CF, et al. Pandemic peak SARS-CoV-2 infection and seroconversion rates in London frontline health-care workers. Lancet. 2020;396(10246):e6-e7.

15. Garcia-Basteiro AL, et al. Seroprevalence of antibodies against SARS-CoV-2 among health care workers in a large Spanish reference hospital. Nat Commun. 2020;11(1):3500

16. Zhou W, et al. First infection by all four non-severe acute respiratory syndrome human coronaviruses takes place during childhood. BMC Infect Dis. 2013;13:433

17. Killerby ME, et al. Human coronavirus circulation in the United States 2014-2017. J Clin Virol. 2018;101:52-56.

18. Ou X, et al. Characterization of spike glycoprotein of SARS-CoV-2 on virus entry and its immune cross-reactivity with SARSCoV. Nat Commun. 2020;11(1):1620.

19. Pinto D, et al. Cross-neutralization of SARS-CoV-2 by a human monoclonal SARS-CoV antibody. Nature. 2020;583(7815):290-295.

20. Sagar M, et al. Recent endemic coronavirus infection is associated with less-severe COVID-19. J Clin Invest. 2021;131(1):143380

21. Mulligan MJ, et al. Phase I/II study of COVID-19 RNA vaccine BNT162b1 in adults. Nature. 2020;586(7830):589-593.

22. Bacher $\mathrm{P}$, et al. Low-avidity CD4 ${ }^{+} \mathrm{T}$ cell responses to SARS-CoV-2 in unexposed individuals and humans with severe COVID-19. Immunity. 2020;53(6):1258-1271

23. Johnson M, et al. Evaluation of a novel multiplexed assay for determining IgG levels and functional activity to SARS-CoV-2 J Clin Virol. 2020;130:104572.

24. Wrapp D, et al. Cryo-EM structure of the 2019-nCoV spike in the prefusion conformation. Science. 2020;367(6483):1260-1263

25. Klumpp-Thomas C, et al. Standardization of ELISA protocols for serosurveys of the SARS-CoV-2 pandemic using clinical and at-home blood sampling. Nat Commun. 2021;12(1):113.

26. FDA. EUA Authorized Serology Test Performance. https://www.fda.gov/medical-devices/coronavirus-disease-2019-covid-19emergency-use-authorizations-medical-devices/eua-authorized-serology-test-performance. Accessed March 15, 2021.

27. Garnett E, et al. Clinical validation and performance evaluation of the automated vitros total anti-SARS-CoV-2 antibodies assay for screening of serostatus in COVID-19. Am J Clin Pathol. 2020;154(6):742-747.

28. Hilpert K, et al. Cellulose-bound peptide arrays: preparation and applications. Biotechnol Genet Eng Rev. 2007;24:31-106

29. DeLano WL, et al. Convergent solutions to binding at a protein-protein interface. Science. 2000;287(5456):1279-1283.

30. Greenland S. Basic methods for sensitivity analysis of biases. Int J Epidemiol. 1996;25(6):1107-1116. 